\title{
Ultra High Vacuum Transmission Electron Microscopy of Metal/2D Material Contacts
}

\author{
Joachim Dahl Thomsen ${ }^{1}$, Kate Reidy ${ }^{1}$, Vera Zarubin ${ }^{1}$ and Frances M. Ross ${ }^{1}$ \\ ${ }^{1}$ Department of Materials Science and Engineering, Massachusetts Institute of Technology, Cambridge, \\ MA, USA.
}

Semiconducting two-dimensional (2D) transition metal dichalcogenides (TMDs) are widely recognized as an alternative to silicon for future transistor devices. The benefit of these materials arises from the strong confinement of charge carriers which greatly reduces leakage currents, leading to lower power consumption. However, practical microelectronic applications of TMDs require electrical contacts to the 2D material with low barriers to the flow of current. Achieving this has proven to be a challenge due to, for example, Schottky barrier formation and Fermi level pinning at the TMD/metal interface [1]. Approaches for improving electrical contacts to TMDs, and more generally other van der Waals materials, include testing a multitude of metals, patterning the 2D material to increase edge contact length to the metal, and ultra-high vacuum deposition methods [2]. Titanium is an interesting starting point for electronic devices that use 2D materials [3] because its properties have been studied in depth for conventional silicon electronics, where it is commonly used as an adhesion layer for metal $\mathrm{Ti} / \mathrm{Au}$ multilayer contacts. The semimetal $\mathrm{Bi}$ is also of interest, since low contact resistance between $\mathrm{Bi}$ and TMDs has been reported [4]. These phenomenological results point to the need for microscopic understanding of the $2 \mathrm{D} /$ metal interface to clarify the relationship between processing conditions, morphology, and contact resistance.

Ultra-high vacuum transmission electron microscopy (UHV-TEM) provides a unique tool for exploring the structure of the 2D/metal interface. We prepare samples of suspended 2D materials such as graphene, $\mathrm{hBN}, \mathrm{MoS}_{2}$ and $\mathrm{WSe}_{2}$ with clean surfaces by transfer using a hydrophobic polymer, cellulose acetate butyrate, onto a silicon chip with a holey silicon nitride layer [5]. Once in the vacuum system, we anneal the sample by Joule heating then deposit metals such as $\mathrm{Ag}, \mathrm{Nb}$, $\mathrm{Ti}, \mathrm{Au}$ and $\mathrm{Bi}$ [6-8]. We find that annealing immediately prior to deposition, which presumably removes adsorbed water, adventitious contamination and polymeric contamination, enables us to obtain large, epitaxial metal grains on the 2D material surface. Further deposition allows these nuclei to coalesce into a continuous film. To create a stack such as $\mathrm{Au} / \mathrm{Ti}$, we deposit $\mathrm{Ti}$, image the sample and then deposit $\mathrm{Au}$, all without breaking vacuum. We can also modify the surface of the 2DM using focused ion beam patterning before metal deposition [9] to explore the effects of surface features on nanocrystal nucleation.

Figure 1 shows a temperature series obtained by depositing Ti under UHV onto a van der Waals surface (here, graphene). The lattices are aligned in each case, with Ti [0001] || Gr [0001] and Ti [01-10] || Gr [1120], but the morphology of the islands is strongly affected by the temperature of the substrate during deposition. In the case of $\mathrm{Au} / \mathrm{Ti}$ stacks, we find that a further control variable is the condition of the $\mathrm{Ti}$ surface before the $\mathrm{Au}$ is deposited. When the sequential deposition steps are carried out under vacuum conditions $\left(10^{-9}\right.$ Torr pressure), large grains of Au form with an epitaxial relationship to the Ti beneath. However, exposure to even $10^{-6}$ Torr oxygen for several minutes after Ti deposition is sufficient to disrupt the epitaxy between $\mathrm{Ti}$ and $\mathrm{Au}$, resulting in small polycrystalline Au islands, presumably due to a surface oxide formed on Ti. The ability to control the background pressure during deposition and imaging is therefore important in evaluating the details of epitaxy. Control of nucleation, coalescence and epitaxy is 
essential if we are to develop strategies to optimise the electrical properties of the metal/2D interface [10].

References:

[1] Y Xu et al, ACS Nano 10 (2016), p. 4895.

[2] CD English et al, Nano Letters 16 (2016), p. 3824.

[3] M Todeschini et al, ACS Applied Materials and Interfaces 9 (2017), p. 37374.

[4] PC Shen et al, Nature 593 (2021), p. 211.

[5] JD Thomsen et al, Physical Review B 96 (2017), p. 1.

[6] JD Thomsen et al, Microscopy and Microanalysis 26 (S2) (2020), p. 1094.

[7] P Periwal et al, Applied Physics Reviews 7 (2020), p. 031402.

[8] K Reidy et al, Nature Communications 12 (2021), p. 1.

[9] V Zarubin et al, Microscopy and Microanalysis 27 (S1) (2021), p. 342.

[10] This work was carried out with the use of facilities and instrumentation supported by NSF through the MIT Materials Research Science and Engineering Center DMR-1419807, and through the use of facilities within MIT.nano. K.R. acknowledges a MIT MathWorks Engineering Fellowship and an OGE MIT Fellowship. J.D.T acknowledges support from Independent Research Fund Denmark though grant number 9035-00006B.
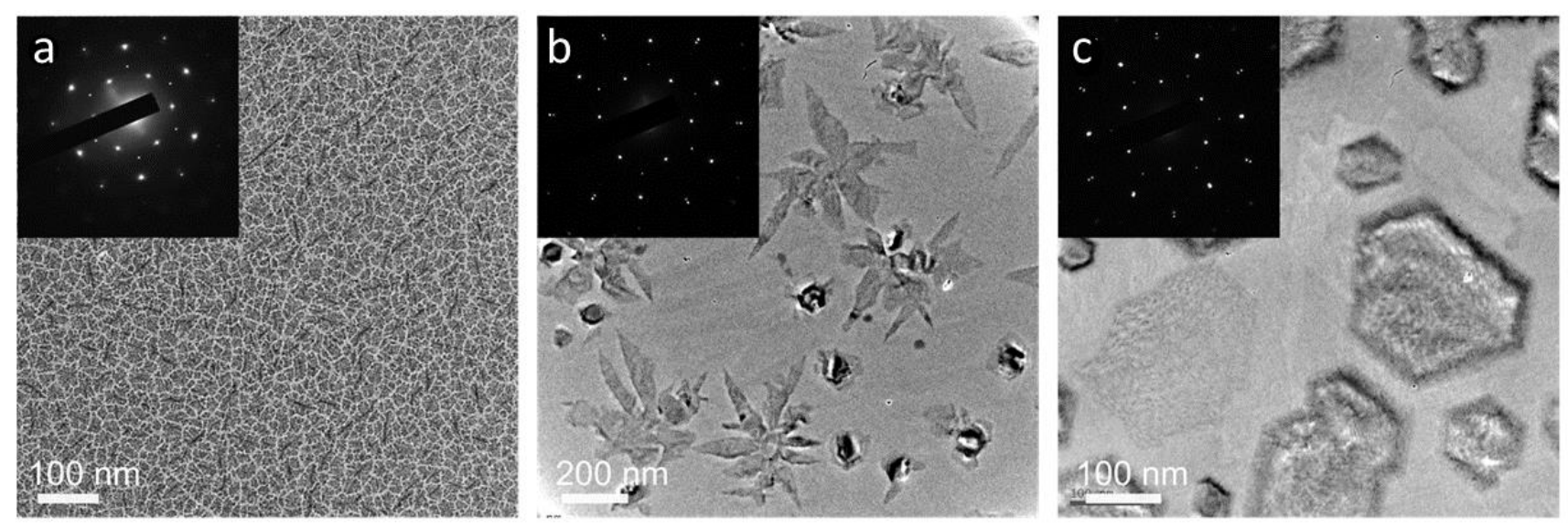

Figure 1. Bright field images and diffraction patterns of a free-standing graphene substrate after deposition of ultra-thin Ti $(<1 \mathrm{~nm})$ under UHV conditions. (a) Substrate at room temperature leading to dendritic yet epitaxial islands. (b) Substrate at $300^{\circ} \mathrm{C}$ leading to larger, single crystal islands. (c) Substrate at $400^{\circ} \mathrm{C}$ leading to compact, faceted, dislocate. 